\title{
Fibrilação Atrial e Tromboembolismo Sistêmico - Relação Causal ou uma Mensagem de Doença Atrial?
}

\author{
Atrial Fibrillation and Systemic Thromboembolism - Causal Correlation or a \\ Message of Atrial Disease?
}

J. Tarcísio Medeiros de Vasconcelos ${ }^{1, *}$, Carlos Eduardo Duarte ${ }^{1}$, Silas dos Santos Galvão Filho ${ }^{1}$

ORCID IDs

\author{
Vasconcelos JTM (D) https://orcid.org/0000-0002-5152-2648 \\ Duarte CE (D) https://orcid.org/0000-0001-6671-0820 \\ Galvão Filho SS (D) https://orcid.org/0000-0001-5236-164X
}

\section{INTRODUÇÃO}

A relação entre fibrilação atrial (FA) e tromboembolismo é conhecida de longa data. Em 1951, Raymond Daley et al. associaram com informações consistentes a ocorrência dessa arritmia a eventos embólicos sistêmicos em pacientes portadores de cardiopatia reumática crônica ${ }^{1}$. Nesse estudo envolvendo 194 portadores de cardiopatia reumática vítimas de tromboembolismo, contando com informações de autópsia em 39 pacientes, ficou demonstrada a presença de FA em cerca de 90\% dos casos. O clássico estudo de Framingham, publicado em 1978, foi o primeiro grande estudo que estabeleceu essa mesma relação em indivíduos não reumáticos, demonstrando que os portadores de FA têm perto de seis vezes mais chances de apresentar um acidente vascular cerebral do que aqueles indivíduos livres de FA com características ajustadas para sexo, idade e pressão arterial ${ }^{2}$. A inclusão de indivíduos reumáticos elevou esse risco para algo em torno de 18 vezes. Desde essa importante publicação, diversos estudos corroboraram esses achados ${ }^{3-7}$.

$\mathrm{Na}$ Tabela 1 são apresentados os resultados de cinco estudos envolvendo mais de 100 mil pacientes, que situam a prevalência de FA em pacientes vítimas de um acidente vascular cerebral isquêmico (AVCi) em torno de 15 a 40\%. É bastante razoável contudo deduzir que, mesmo nos 60 a 85\% dos pacientes que apresentam um AVCi sem o diagnóstico pregresso de FA, a prevalência da arritmia seja maior, considerando a possibilidade de arritmia assintomática não diagnosticada. Em revisão sistemática e metanálise de 32 estudos envolvendo cerca de 5.000 pacientes sem antecedentes de FA que sofreram um AVCi submetidos a monitoramento agressivo do ritmo cardíaco, por Holter de 24 h, Holter de 7 dias ou monitor de eventos implantável, demonstrou-se o diagnóstico de FA em cerca de $11 \%$ dos pacientes ${ }^{8}$. Portanto, a partir das informações obtidas nesses grandes estudos ao longo das últimas seis décadas, com resultados incorporados de significativa força e consistência de associação, o seguinte paradigma foi construído: "fibrilação atrial causa AVCi tromboembólico". 
Tabela 1. Resultados de cinco diferentes estudos que juntos incluem mais de 100 mil pacientes que caracterizam a grande frequência de fibrilação atrial em pacientes vítimas de acidente vascular cerebral isquêmico.

\begin{tabular}{|c|c|c|c|}
\hline Autor & Ano & $\begin{array}{l}\text { Acidente vascular cerebral } \\
\text { isquêmico (n) }\end{array}$ & Fibrilação atrial (\%) \\
\hline Wolf PA et al. ${ }^{3}$ & 1987 & 462 & 14,7 \\
\hline Thygesen SK et al. ${ }^{4}$ & 2009 & 3.849 & 19,2 \\
\hline Hannon $\mathrm{N}$ et al..$^{5}$ & 2010 & 568 & 31,2 \\
\hline Björck S et al. ${ }^{6}$ & 2013 & 4.565 & 38 \\
\hline Friberg L et al. ${ }^{7}$ & 2014 & 94.083 & 33,4 \\
\hline
\end{tabular}

\section{FIBRILAÇÃO ATRIAL E DOENÇA ATRIAL}

A FA identificada como um fator de risco independente para AVCi passou a ter na terapia anticoagulante uma pedra angular do seu tratamento, com resultados consistentemente positivos quando outros elementos de estratificação de risco são agregados, que implicaram na construção de escores de risco, hoje universalmente empregados para tomada de decisão terapêutica, tais como $\mathrm{CHADS}_{2}, \mathrm{CHA}_{2} \mathrm{DS}_{2}-\mathrm{VASc}$ e ATRIA ${ }^{9-11}$. É interessante refletir sobre o condicionamento de risco da FA para o AVCi à agregação de outros elementos paralelos de catalisação desse risco, tais como presença ou ausência de insuficiência cardíaca, hipertensão, diabetes, idade avançada e doença valvar. Também é interessante notar que esses mesmos elementos de risco para ocorrência de AVCi no paciente portador de FA também são identificados como elementos indicativos de risco para a própria ocorrência de FA futura em pacientes sem FA documentada.

Em um grande estudo populacional sueco, a prevalência de FA em pacientes com um escore $\mathrm{CHA}_{2} \mathrm{DS}_{2}-\mathrm{VASc}$ igual a zero era um pouco maior que 10\%, chegando a valores próximos a $70 \%$ quando esse escore chegava a um valor igual a nove 7 . Por que a FA isolada encerra uma chance de risco pequena para AVCi em comparação àquela em que ocorre em associação ao diabetes e à hipertensão por exemplo? Seria a FA um fator diretamente determinante do AVCi? Ou seria ela apenas a expressão de um risco cardioembólico diferenciado ligado a uma condição de base trombogênica subjacente? De encontro a esses questionamentos vêm dados que indicam que o emprego do escore $\mathrm{CHA}_{2} \mathrm{DS}_{2}-\mathrm{VASc}$ identifica o risco de tromboembolismo também de indivíduos em ritmo sinusal. Em um grande estudo dinamarquês envolvendo mais de 136 mil indivíduos portadores de insuficiência cardíaca em ritmo sinusal, o emprego desse escore foi capaz de identificar de forma bastante previsível o risco tromboembólico nas diferentes categorias de estratificação ${ }^{12}$. Em outro estudo comunitário realizado em Trieste na Itália, no qual foram incluídos perto de 12,5 mil indivíduos hipertensos sem uso de terapia anticoagulante, com cerca de 11 mil indivíduos em ritmo sinusal e 1,5 mil portadores de fibrilação atrial, o escore $\mathrm{CHA}_{2} \mathrm{DS}_{2}-\mathrm{VASc}$ também identificou risco tromboembólico nas diferentes categorias de estratificação nos dois grupos de pacientes; de forma surpreendente, a capacidade de identificação de risco tromboembólico em pacientes com escore igual ou maior que seis foi equivalente entre pacientes com fibrilação atrial e ritmo sinusa $1^{13}$. Portanto há elementos indicativos de que um escore originalmente desenvolvido e validado para estratificar risco de tromboembolismo em pacientes portadores de fibrilação atrial é também capaz de identificar o risco de desenvolvimento futuro da própria FA e o risco tromboembólico em indivíduos com ritmo sinusal estável, ou seja, sem FA.

$\mathrm{O}$ desdobramento do escore $\mathrm{CHA}_{2} \mathrm{DS}_{2}$-VASc em seus diversos componentes permite o desenvolvimento de um raciocínio que coloca os achados descritos dentro de um modelo de plausibilidade biológica. Cardiopatia, insuficiência cardíaca, hipertensão, idade avançada, diabetes e doença vascular são fatores determinantes ou marcadores de agressão miocárdica atrial. A agressão miocárdica atrial é potencialmente trombogênica, sendo determinante e/ou catalisadora da própria FA, que por sua vez retroalimenta a própria agressão miocárdica (Figs. 1 e 2). Entra-se assim em contato com o conceito de cardiopatia fibrótica atrial. 


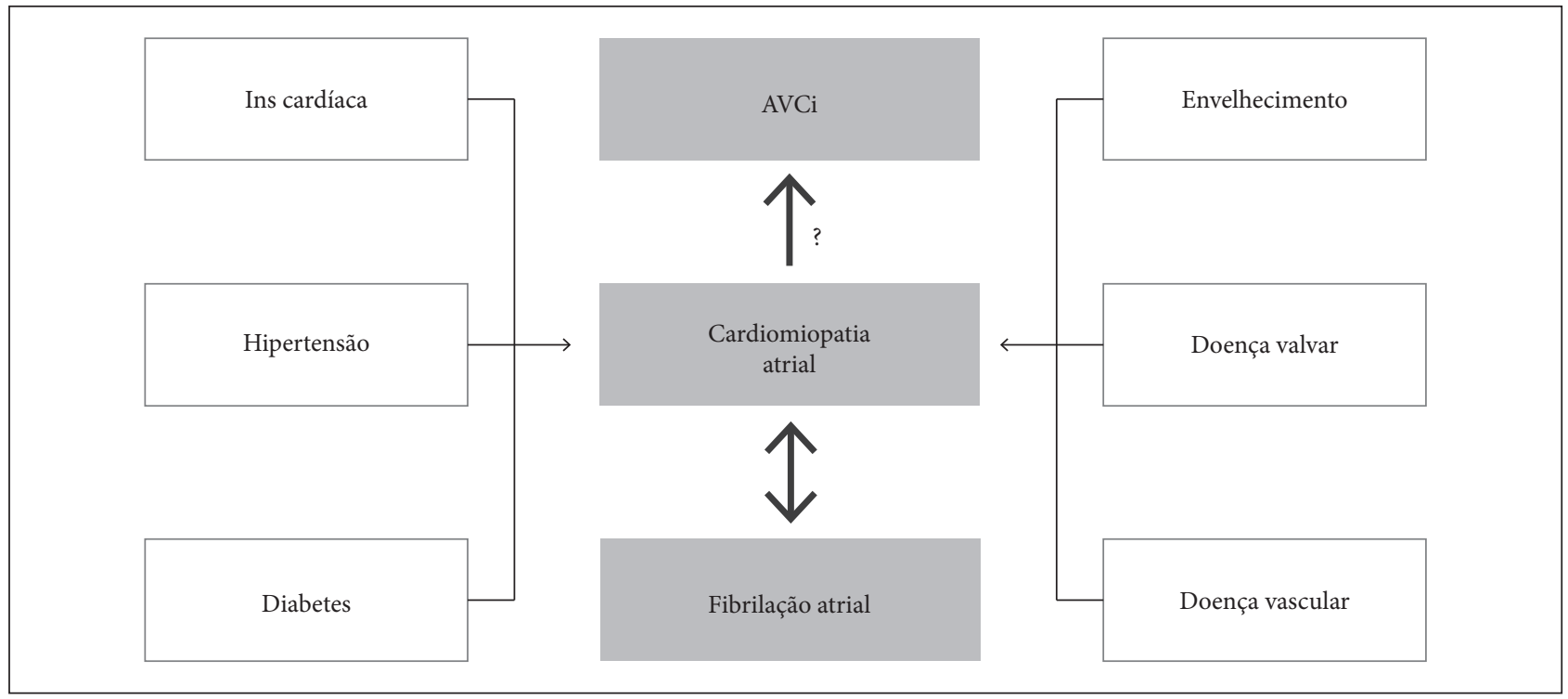

Figura 1. Potencial relação dos marcadores de risco tromboembólico da fibrilação atrial com o próprio acometimento estrutural e elétrico atrial. Condições como insuficiência cardíaca, hipertensão, diabetes, envelhecimento, doença valvar e doença vascular são de fato potenciais fatores determinantes de agressão estrutural ao miocárdio atrial implicando em uma cardiomiopatia atrial. A cardiomiopatia atrial é determinante de fibrilação atrial que por sua vez realimenta o processo de agressão miocárdica atrial. O risco cardioembólico seria na verdade provocado não pela fibrilação atrial diretamente (que pode estar ausente), mas sim pela doença atrial estabelecida.

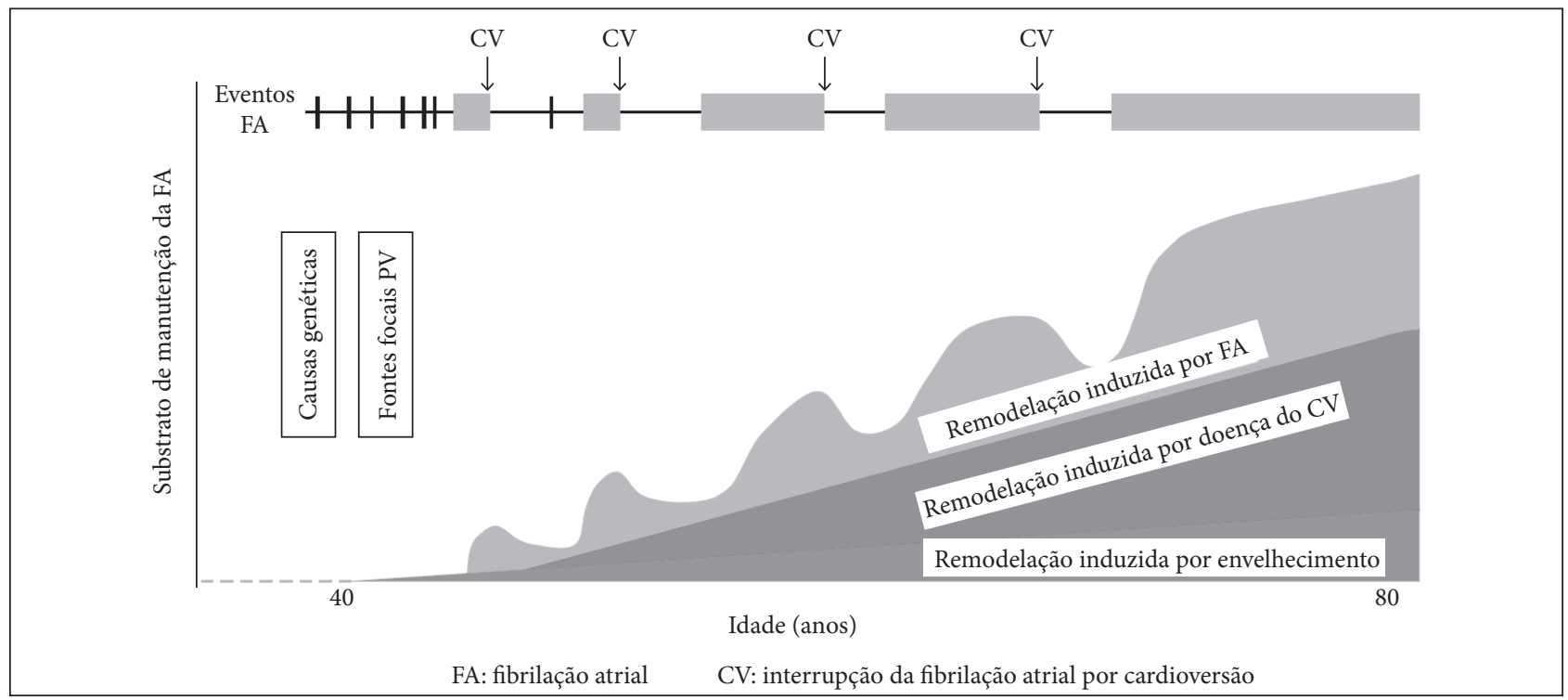

Figura 2. Inter-relação de fatores determinantes de remodelamento elétrico e estrutural atrial. A fibrilação atrial pode ser primária ligada a fatores genéticos e a focos disparadores pulmonares ou pode ser secundária à própria doença atrial progressiva fruto do envelhecimento e/ou do acometimento muscular secundário a comorbidades. Tem-se um cenário de combinação de fatores cujo resultado final é a cardiomiopatia fibrótica atrial. Adaptado de Guichard e Nattel ${ }^{14}$.

A caracterização de cardiopatia fibrótica atrial foi originalmente feita em 2012 por Kottkamp, que chamou a atenção para pacientes que apresentavam acometimento estrutural e eletrofisiológico da musculatura atrial na ausência de doenças subjacentes que pudessem justificá-las ${ }^{15}$. A Fig. 3 corresponde aos mapas de voltagem dos átrios direito e esquerdo obtidos de uma mulher de 42 anos de idade, sem doença estrutural cardíaca estabelecida, portadora de taquicardia e fibrilação atrial "solitárias". As imagens foram obtidas durante estudo eletrofisiológico e ablação percutânea para tratamento dessas arritmias. As áreas na cor cinza correspondem às regiões de baixa voltagem, indicativas da presença de cicatriz e as áreas na cor roxa às regiões de miocárdio normal. Notam-se cicatrizes extensas ao longo das paredes de ambos os átrios, com miocárdio normal em áreas restritas, indicando importante acometimento fibrótico biatrial. Em uma circunstância assim, mesmo tratando-se de uma 
paciente com baixo risco cardioembólico por critérios clássicos $\left(\mathrm{CHA}_{2} \mathrm{DS}_{2}-\mathrm{VASC}=0\right)$, é natural o questionamento de utilização permanente de terapia anticoagulante, independentemente da presença ou não de ritmo sinusal estável.

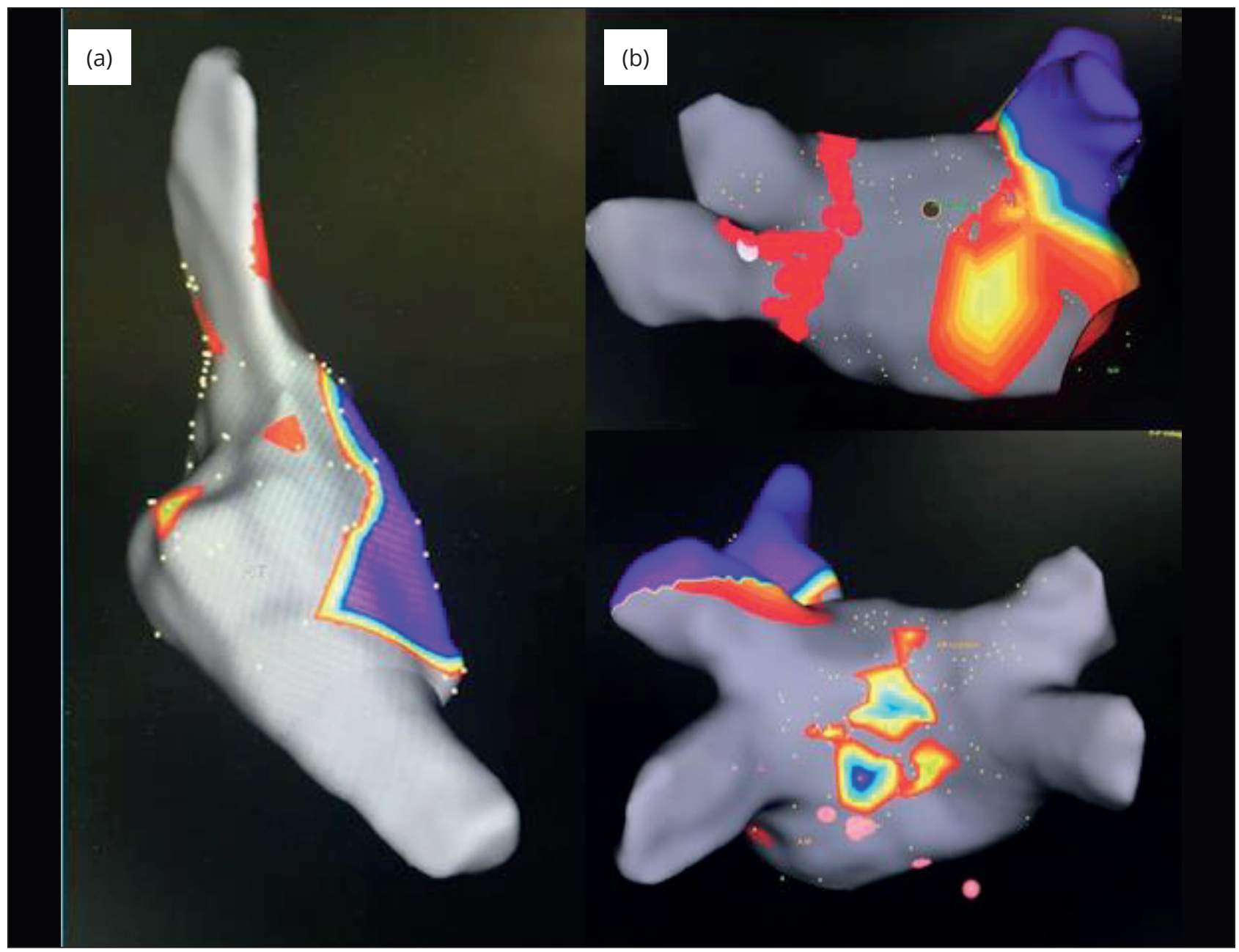

Figura 3. Mapas de voltagem dos átrios direito (a) e esquerdo (b) construídos durante procedimento de ablação.

Em um excelente documento de consenso elaborado por quatro sociedades médicas internacionais, no qual uma grande revisão sobre o tema foi realizada, publicado em 2016, a cardiomiopatia fibrótica atrial foi classificada em quatro tipos conforme o padrão de acometimento histológico, relacionados aos diferentes tipos de fatores etiológicos determinantes de agressão muscular ${ }^{16}$. O conceito e caracterização da doença ficou nesse documento mais abrangente e detalhado, não restrito apenas ao acometimento atrial dissociado da identificação de doenças sistêmicas, mas incluído dentro do universo de todas as doenças que podem gerar alguma forma de agressão miocárdica atrial, incluindo envelhecimento, obesidade, hipertensão, miocardite, insuficiência cardíaca, diabetes, doenças de depósito, doenças hereditárias, remodelamento induzido pela própria arritmia etc. A questão fundamental é se a presença do acometimento estrutural atrial não é o principal elemento determinante de um risco cardioembólico aumentado, sendo a fibrilação atrial um simples marcador da presença de doença e/ou um mero agravante desse risco cardioembólico. Diversas informações apontam nesse sentido. A presença de dilatação atrial tem sido identificada como fator de risco para ocorrência de AVCi mesmo na ausência de fibrilação atrial diagnosticada ${ }^{17}$. A presença de fibrose atrial diagnosticada por técnica de realce tardio na ressonância magnética em alguns estudos foi relacionada a uma história pregressa de $\mathrm{AVCi}$, presença de trombo em apêndice atrial esquerdo e contraste espontâneo em átrio esquerdo diagnosticados por ecocardiograma transesofágico ${ }^{18-20}$. O diagnóstico de fibrose atrial por mapa de voltagem em procedimentos de ablação para tratamento de fibrilação atrial foi relacionado a uma maior probabilidade pregressa à ablação, da ocorrência de $\mathrm{AVCi}$ e lesão cerebral silenciosa diagnosticada por ressonância magnética ${ }^{21}$. 


\section{ACIDENTE VASCULAR CEREBRAL E RITMO CARDÍACO: A DOCUMENTAÇÃO ELETROCARDIOGRÁFICA DO EVENTO}

O cenário ideal para estabelecer uma relação clara de causa e efeito entre a fibrilação atrial e o evento cardioembólico é a documentação do próprio evento cardioembólico mediante monitoramento contínuo do ritmo cardíaco, em especial no período precedente à sua ocorrência. O desafio natural para obtenção dessas informações obviamente é a disponibilização de monitoramento cardíaco antes e durante o momento de acontecimento do evento cardioembólico. Os registros do ritmo cardíaco subjacente em vítimas de um acidente tromboembólico habitualmente se dá após a sua ocorrência, situações em que a presença de ritmo sinusal estável não significa ritmo normal nos momentos, dias ou semanas que antecederam o próprio evento. É absolutamente plausível imaginar uma sequência de fatos iniciada pela ocorrência de fibrilação atrial precipitando a formação de um trombo atrial esquerdo, seu deslocamento gerando uma complicação embólica à distância e conversão espontânea da fibrilação atrial a ritmo sinusal, de tal modo que, por ocasião do primeiro atendimento médico, a presença de ritmo sinusal seja constatada.

A possibilidade de monitoramento do ritmo cardíaco por ultralonga duração com dispositivos implantáveis abriu uma nova perspectiva de investigação que permite um aprofundamento nesse tema. Os dispositivos cardíacos de estimulação, incluindo marcapassos, cardiodesfibriladores e ressincronizadores cardíacos são próteses que possuem sofisticados recursos de monitoramento permanente do ritmo cardíaco e armazenamento dessas informações, com a vantagem de permitirem o registro dos eventos atrial e ventricular intracardíacos agregando assim inestimável precisão diagnóstica (Fig. 4).

\begin{tabular}{|c|c|c|c|}
\hline \multicolumn{3}{|c|}{$\begin{array}{l}\text { Device: Ensura DR MRI EN1DR01 } \\
\text { Serial Number: PZW643376S } \\
\text { Monitored AT/AF Episode \#554 } \\
\text { Episode \#554: 27-Nov-2019 22:33:49 }\end{array}$} & \\
\hline \multicolumn{4}{|l|}{ Episode Summary } \\
\hline $\begin{array}{l}\text { Initial type } \\
\text { Duration } \\
\text { A/V Max Rate } \\
\text { A. Median } \\
\text { Activity at onset }\end{array}$ & \multicolumn{3}{|c|}{$\begin{array}{l}\text { AT/AF Monitor (spontaneous) } \\
11 \mathrm{~min} \\
300 \mathrm{bpm} / 107 \mathrm{bpm} \\
261 \mathrm{bpm}(230 \mathrm{~ms}) \\
\text { Rest. Sensor }=79 \mathrm{bpm}\end{array}$} \\
\hline Parameter Settings & Zones & \multirow{2}{*}{\multicolumn{2}{|c|}{$\frac{\text { A. Interval (rate) }}{350 \mathrm{~ms}(171 \mathrm{bpm})}$}} \\
\hline Monitor & 1 & & \\
\hline EGM Source & Range & \multicolumn{2}{|c|}{ Sensitivity } \\
\hline $\begin{array}{ll}\text { EGM1 } & \text { Atip to Aring } \\
\text { EGM2 } & \text { RVtip to RVring }\end{array}$ & $\begin{array}{l} \pm 8 \mathrm{mV} \\
\pm 8 \mathrm{mV}\end{array}$ & $\begin{array}{l}\text { Atria } \\
\text { RV }\end{array}$ & $\begin{array}{l}0.3 \mathrm{mV} \\
0.3 \mathrm{mV}\end{array}$ \\
\hline
\end{tabular}

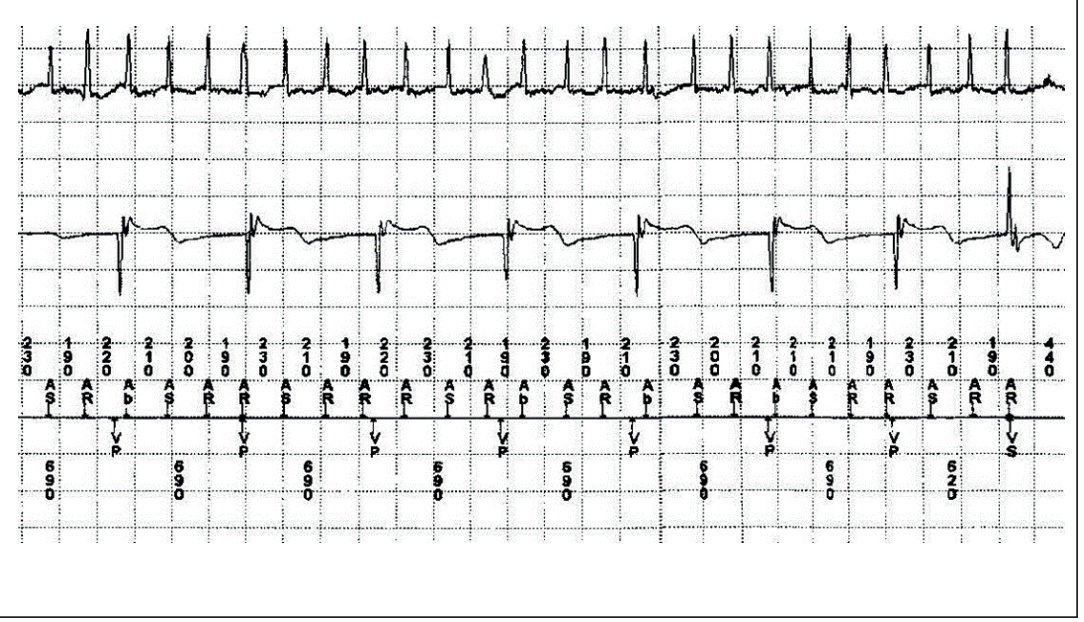

Figura 4. Episódio de taquicardia atrial primária subclínica com 11 minutos de duração, detectada por um marcapasso de dupla-câmara. O primeiro canal corresponde aos eletrogramas atriais, onde fica evidente uma taquicardia atrial com frequência próxima a $300 \mathrm{bpm}$. O segundo canal corresponde aos eletrogramas ventriculares que demonstram que os ventrículos estão sob estimulação do marcapasso no momento da taquicardia, em decorrência de um bloqueio atrioventricular. O canal inferior representa a identificação em marcas de como o dispositivo está interpretando o ritmo cardíaco subjacente.

De fato, alguns estudos têm aproveitado esses recursos tecnológicos com o intuito de avaliar de forma mais consistente as prováveis inter-relações entre a ocorrência de taquicardias atriais e os eventos tromboembólicos em uma perspectiva temporal, com resultados intrigantes. O TRENDS Study ${ }^{22}$, publicado em 2009, foi um estudo observacional prospectivo que recrutou pacientes portadores de dispositivos de estimulação/desfibrilação, com um ou mais fatores de risco cardioembólico (insuficiência cardíaca, hipertensão arterial, diabetes, idade igual ou superior a 65 anos, antecedentes de acidente tromboembólico), para serem acompanhados por um longo período de tempo buscando identificar eventos tromboembólicos nesse seguimento e estabelecer sua relação com o ritmo cardíaco subjacente. O objetivo do estudo na verdade era determinar se episódios subclínicos breves de taquicardias atriais (frequentemente detectados em avaliações corriqueiras de dispositivos) estariam ou não relacionados com um risco 
cardioembólico diferenciado. A premissa seria que pacientes com maior carga de taquicardias atriais, ou seja, com mais taquicardias atriais no período (horas em taquicardia atrial por dia) poderiam corresponder a uma subpopulação de maior risco tromboembólico. Foram consideradas taquicardias atriais relevantes aquelas com duração igual ou superior a 20 segundos. Embora a não inclusão de pacientes anticoagulados fosse prevista no desenho original do estudo, todos os pacientes foram incluídos na avaliação final, provavelmente por restrições no tamanho da amostra aliadas a uma baixa taxa final de desfechos, o que implicaria em uma limitação na valorização de resultados. Perto de 2.500 pacientes foram incluídos no estudo, com acompanhamento de cerca de 1,5 anos. O escore CHADS2 médio foi de 2,2 gerando um risco anualizado superior a 4\% para ocorrência de tromboembolismo sistêmico. Quarenta desfechos ocorreram no seguimento, gerando uma taxa anualizada de eventos de apenas 1,2\%, aquém da esperada para uma população com fibrilação atrial clinicamente diagnosticada com expectativa de risco semelhante. Uma relação direta da carga de taquicardias atriais e a ocorrência de tromboembolismo sistêmico foi demonstrada; pacientes com carga igual ou superior a 5,5 h (correspondente à soma da duração de todas as taquicardias atriais ocorridas em pelo menos 1 dia em uma janela de 30 dias precedentes de monitoramento contínuo) tiveram cerca de duas vezes mais chances de apresentar um evento do que aqueles que tinham carga nula. Em outras palavras, maior o número e/ou duração de taquicardias atriais, maior foi a incidência de desfechos. Contudo um inesperado lapso temporal foi claramente demonstrado entre a ocorrência de taquicardias atriais e o evento tromboembólico: apenas algo em torno de $28 \%$ dos pacientes vítimas de um evento tiveram algum tipo de taquicardia atrial nos 30 dias anteriores a esse evento ${ }^{23}$.

Um estudo mais recente, o ASSERT (Asymptomatic Atrial Fibrillation and Stroke Evaluation in Pacemaker Patients and the Atrial Fibrillation Reduction Atrial Pacing Trial ${ }^{24}$ trouxe resultados semelhantes. Cerca de 2.600 pacientes portadores de marcapassos ou cardiodesfibriladores, sem antecedentes de fibrilação atrial conhecida, com idade maior ou igual a 65 anos, sem uso de anticoagulantes, foram acompanhados com o intuito de avaliar a relação temporal entre a ocorrência de taquicardias atriais e eventos tromboembólicos. Taquicardias atriais foram definidas mediante sua ocorrência detectada nos dispositivos com uma duração superior a 6 minutos. Após um seguimento médio de 30 meses, 51 pacientes foram vítimas de eventos potencialmente cardioembólicos cerebrais e sistêmicos, nos quais taquicardias atriais com mais de 6 minutos de duração foi identificada em 26 casos (51\%). Entretanto, a ocorrência dessas taquicardias atriais se deu em apenas 4 pacientes (8\%) nos 30 dias que antecederam o evento cardioembólico.

Outro estudo multicêntrico, o IMPACT study ${ }^{25}$, avaliou o papel da anticoagulação ocasional na prevenção de acidentes tromboembólicos, baseado na deteç̧ão de taquicardias atriais por cardiodesfibriladores implantáveis. A deteç̧ão de taquicardias atriais pelos dispositivos era notificada rapidamente ao médico assistente por um sistema de monitoramento remoto permanente à distância, possibilitando a administração de fármacos anticoagulantes de maneira intermitente. Antagonistas da vitamina $\mathrm{K}$ e anticoagulantes de ação direta foram utilizados. A hipótese era que a anticoagulação intermitente empregada apenas após o diagnóstico de taquicardias atriais seria uma estratégia superior à anticoagulação permanente baseada em critérios clássicos, na redução de um desfecho composto por acidente vascular cerebral, embolia sistêmica e sangramento maior. Taquicardias atriais eram consideradas relevantes se 36 ou mais ciclos atriais em uma janela de 48 ciclos tivessem frequência superior a 200 bpm. Pacientes com fibrilação atrial permanente foram excluídos. Foi um estudo prospectivo, em que perto de 2.700 pacientes foram randomizados para anticoagulação intermitente ou para o grupo controle. A anticoagulação nos pacientes do grupo controle era instituída de forma permanente, caso fibrilação atrial fosse detectada nas avaliações clínicas presenciais periódicas, seja por métodos convencionais ou pela própria interrogação dos dispositivos. O estudo tinha uma previsão de duração de 36 meses, mas foi interrompido prematuramente aos 24 meses por não se conseguir demonstrar superioridade da anticoagulação intermitente. Sessenta e nove eventos tromboembólicos foram documentados em toda a população; em 58\% (40 eventos) na ausência de taquicardias atriais detectadas em todo o seguimento. Dos 29 pacientes em que taquicardias atriais foram registradas, em 20 a sua ocorrência se deu antes do acidente tromboembólico, dos quais apenas 6 (8,7\%) aconteceram no momento ou nos 30 dias anteriores à ocorrência do acidente tromboembólico. Mais uma vez ficou demonstrada a ausência de uma clara relação temporal entre o evento arrítmico e o evento tromboembólico.

Os resultados desses três estudos estão representados em forma de gráfico na Fig. 5. 


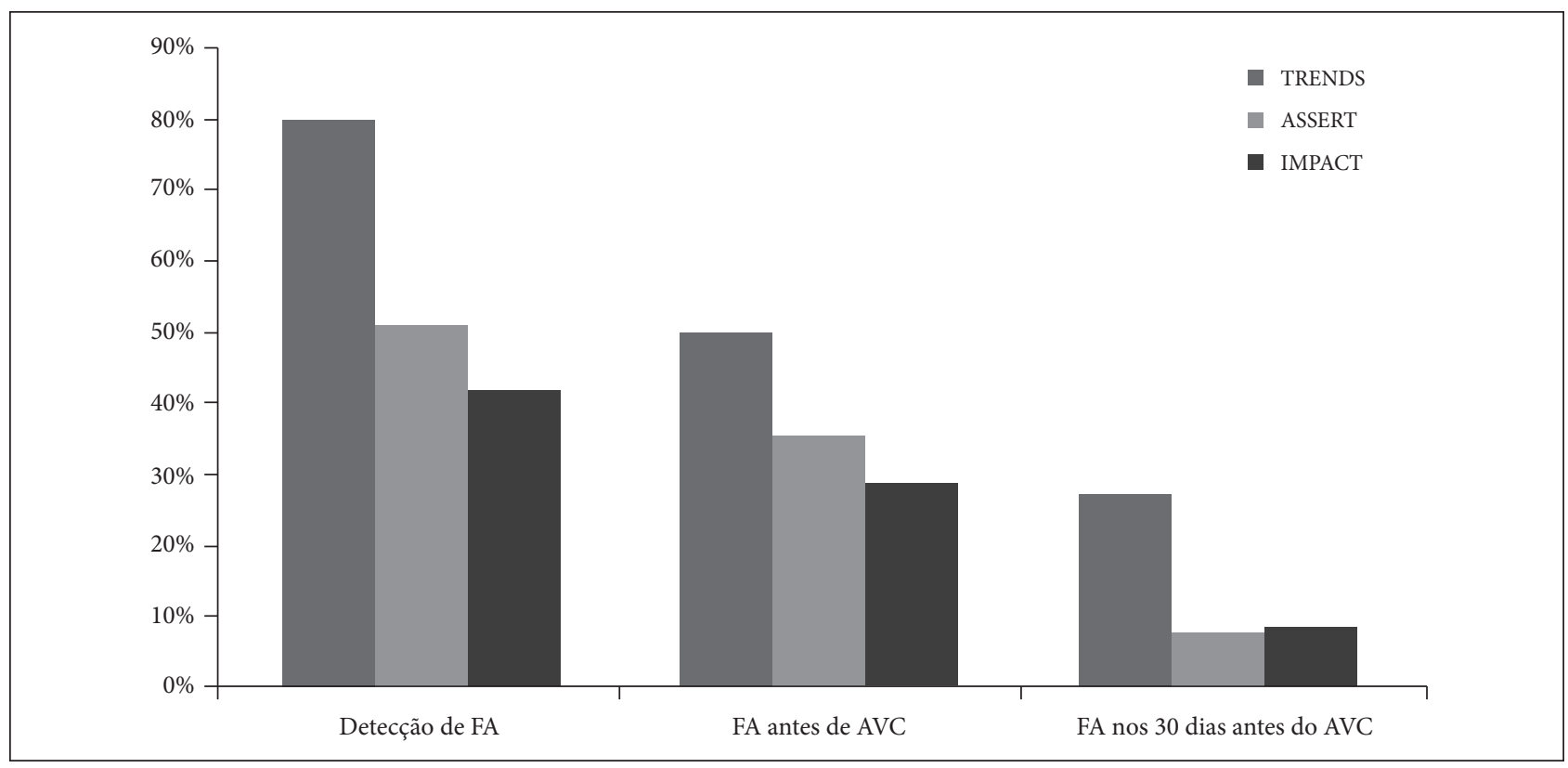

Figura 5. Apresentação gráfica dos resultados dos estudos TRENDS, ASSERT e IMPACT. As informações são derivadas dos pacientes que sofreram acidentes cardioembólicos, predominantemente $\mathrm{AVCi}$, enquanto se encontravam sob monitoramento permanente do ritmo cardíaco. As colunas da esquerda representam os pacientes que em algum momento do monitoramento apresentaram taquicardias atriais. Nota-se que 40 a $80 \%$ dos pacientes tiveram taquicardias registradas. As colunas do meio representam os pacientes que apresentaram taquicardias atriais anteriores ao acidente embólico, variando entre 30 e $50 \%$. As colunas da direita representam os pacientes que apresentaram taquicardias atriais nos 30 dias que antecederam o evento embólico. Nota-se que esse número não atinge $30 \%$.

Uma metanálise recente, publicada em 2018, incluiu 11 estudos que avaliaram o diagnóstico e o significado das taquicardias atriais subclínicas em portadores de dispositivos cardíacos eletrônicos implantáveis ${ }^{26}$. Taquicardias atriais subclínicas tipo fibrilação atrial, foram identificadas em $35 \%$ dos pacientes. A presença de fibrilação atrial subclínica foi fortemente relacionada à ocorrência de fibrilação atrial clínica com um risco cerca de seis vezes maior. Apenas 17\% dos AVCi ocorreram na vigência de fibrilação atrial e apenas $29 \%$ dos pacientes vítimas de acidente cardioembólico tiveram fibrilação atrial registrada pelos dispositivos nos 30 dias anteriores ao evento.

Obviamente, esse conjunto de informações indica que apesar da presença de taquicardias atriais, em especial a fibrilação atrial, ser marcadora de risco para a ocorrência de fenômenos tromboembólicos, não seria a sua própria ocorrência a causa direta do evento em pacientes portadores de taquicardias atriais silenciosas detectadas em dispositivos de estimulação e/ou desfibrilação cardíaca. Entretanto os dados derivados desses estudos devem ser vistos com cuidado em função de uma série de fatores: 1) esses estudos envolveram uma subpopulação específica de pacientes, portadores de marcapassos ou cardiodesfibriladores implantados pela presença de condições clínicas particulares e específicas, não podendo-se transportar essas conclusões para todo o conjunto de indivíduos com fibrilação atrial; 2) as taquicardias atriais diagnosticadas nesses estudos, incluindo a própria fibrilação atrial, corresponderam em geral a taquicardias subclínicas, autolimitadas, em sua maioria de curta duração, cujo significado é potencialmente distinto daquele observado nos pacientes classicamente envolvidos nos grandes estudos que identificaram a fibrilação atrial como uma grande vilã - um dos elementos mais emblemáticos dessa constatação corresponde ao fato de que as taxas de eventos globais nesses pacientes foi caracteristicamente mais baixa do que a esperada para pacientes portadores de fibrilação clinicamente diagnosticada, considerando as classes de risco por escores CHADS $_{2}$ ou $\mathrm{CHA}_{2} \mathrm{DS}_{2}$-VASC; 3 ) deve-se sempre ter em mente as próprias características diagnósticas dos dispositivos cardíacos eletrônicos implantáveis, que são falíveis, em consequência de uma diversidade de fatores, tais como "under ou oversensing" (Fig. 6) ou a própria forma de programação (pré-determinada) para identificação de taquicardias - resumidamente, os dispositivos podem diagnosticar erroneamente taquicardias que não existiram ou deixar de diagnosticar taquicardias que ocorreram de fato. 


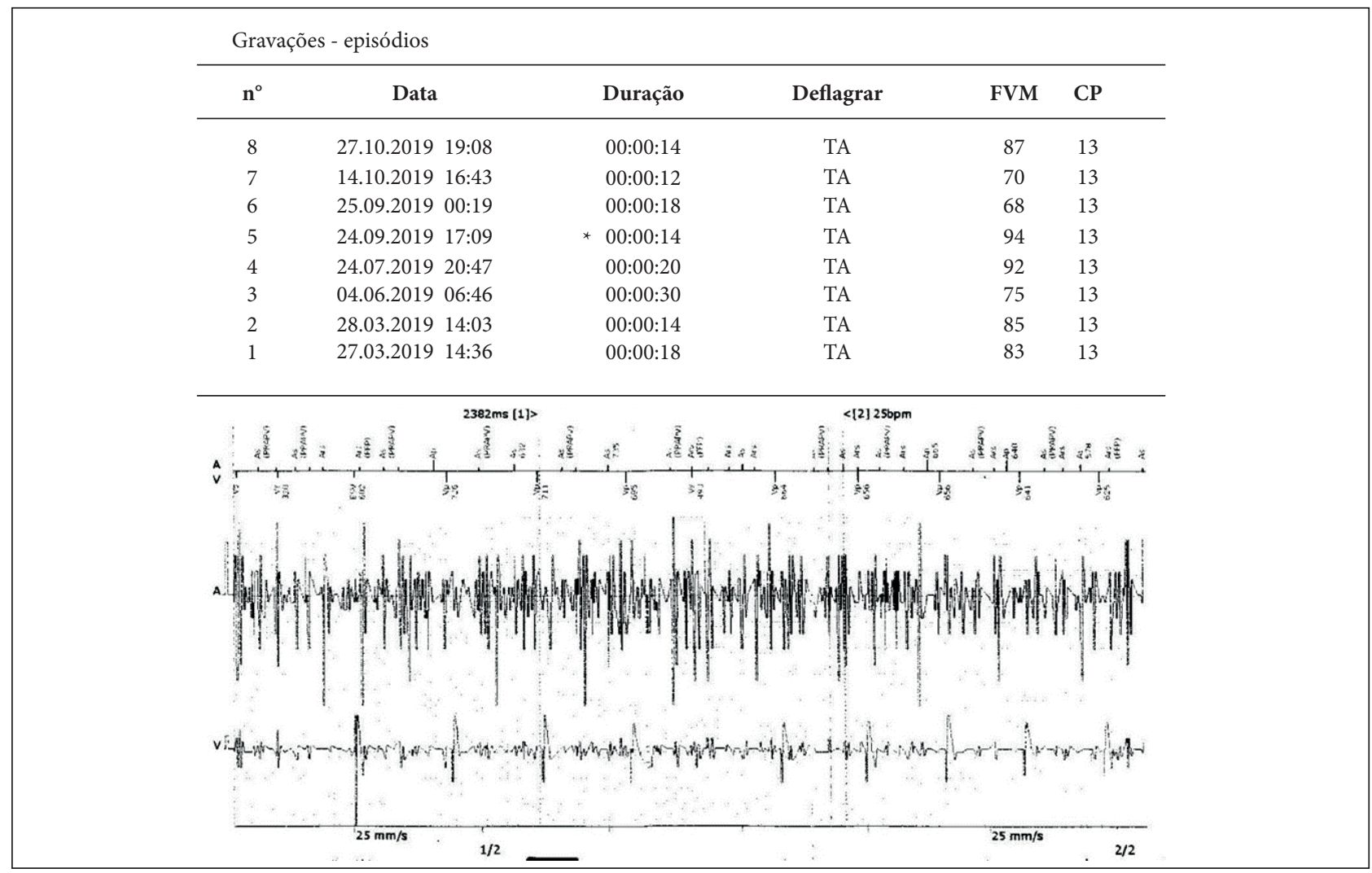

Figura 6. Relatório obtido de uma avaliação de dispositivo que informa a ocorrência de diversos episódios de taquicardia atrial. A análise dos eletrogramas intracardíacos, contudo, demonstra a presença de artefatos elétricos nos canais atrial e ventricular correspondentes a ruídos elétricos possivelmente consequentes a fraturas incompletas dos eletrodos. Trata-se, portanto, de uma falsa taquicardia atrial.

\section{PARA ONDE IREMOS?}

Diante de todas as considerações anteriormente feitas, o que fica caracterizado no mínimo, é que átrios doentes "fibrilam", fibrilação produz ou agrava doença atrial; que fatores classicamente apontados como determinantes de risco para tromboembolismo na fibrilação atrial são também (ou antes disso) fatores de risco para desenvolvimento ou presença de doença atrial e para a própria ocorrência de fibrilação atrial futura; e que a relação direta de causa e efeito entre fibrilação atrial e a ocorrência pontual do evento embólico é duvidosa. Sob o aspecto diagnóstico, abre-se o cenário de investigação mais agressiva dos pacientes, seja em ritmo sinusal seja em fibrilação atrial, no sentido de identificar elementos que permitam reconhecer a presença de cardiopatia fibrótica atrial. Sob os aspectos profilático e terapêutico, abre-se o cenário de prevenir a doença atrial por abordagem das condições periféricas determinantes, tratar primariamente a doença atrial estabelecida pelo tratamento das doenças determinantes, manter o ritmo sinusal para evitar o agravamento da própria doença atrial e ampliar a perspectiva da determinação do risco cardioembólico para além daquilo que fazemos no momento.

É provável que a presença ou ausência de "doença atrial" potencialmente trombogênica no futuro seja o ponto de partida para a estratificação de risco cardioembólico e não o simples diagnóstico de fibrilação atrial, o que deverá levar ao desenvolvimento de novos escores de risco que incluam no seu escopo "estrutura e função atrial".

\section{REFERÊNCIAS}

1. Daley R, Mattingly TW, Holt CL, Bland EF, White PD. Systemic arterial embolism in rheumatic heart disease. Am Heart J. 1951;42(4):566-81. https://doi.org/10.1016/00028703(51)90152-4
2. Wolf PA, Dawber TR, Thomas E, Thomas Junior HE, Kannel WB. Epidemiologic assessment of chronic atrial fibrillation and risk of stroke: The Framingham Study. Neurology. 1978;28(10):973-7. https://doi.org/10.1212/wnl.28.10.973 
3. Wolf PA, Abbott RD, Kannel WB. Atrial fibrillation: a major contributor to stroke in the elderly. The Framingham Study. Arch Intern Med. 1987;147(9):1561-4.

4. Thygesen SK, Frost L, Eagle KA, Johnsen SP. Atrial fibrillation in patients with ischemic stroke: A population-based study. Clin Epidemiol. 2009;1:55-65. https://doi.org/10.2147/clep.s4794

5. Hannon N, Sheehan O, Kelly L, Marnane M, Merwick A, Moore A, et al. Stroke associated with atrial fibrillationincidence and early outcomes in the north Dublin population stroke study. Cerebrovasc Dis. 2010;29(1):43-9. https://doi. org/10.1159/000255973

6. Björck S, Palaszewski B, Friberg L, Bergfeldt L. Atrial fibrillation, stroke risk, and warfarin therapy revisited: a population-based study. Stroke. 2013;44(11):3103-8. https://doi.org/10.1161/ STROKEAHA.113.002329

7. Friberg L, Rosenqvist $M$, Lindgren A, Terént A, Norrving B, Asplund K. High prevalence of atrial fibrillation among patients with ischemic stroke. Stroke. 2014;45(9):2599-605. https://doi. org/10.1161/STROKEAHA.114.006070

8. Kishore A, Vail A, Majid A, Dawson J, Lees KR, Tyrrell PJ, et al. Detection of atrial fibrillation after ischemic stroke or transient ischemic attack - A systematic review and metaanalysis. Stroke. 2014;45(2):520-6. https://doi.org/10.1161/ STROKEAHA.113.003433

9. Gage BF, Waterman AD, Shannon W, Boechler M, Rich MW, Radford MJ. Validation of clinical classification schemes for predicting stroke. Results from the national registry of atrial fibrillation. JAMA. 2001;285(22):2864-70. https://doi. org/10.1001/jama.285.22.2864

10. Lip GYH, Nieuwlaat R, Pisters R, Lane DA, Crijns HJ. Refining clinical risk stratification for predicting stroke and thromboembolism in atrial fibrillation using a novel risk factor-based approach. The euro heart survey on atrial fibrillation. CHEST. 2010;137(2):26372. https://doi.org/10.1378/chest.09-1584

11. Singer DE, Chang Y, Borowsky LH, Fang MC, Pomernacki NK, Udaltsovaet $N$, et al. A new risk scheme to predict ischemic stroke and other thromboembolism in atrial fibrillation: The ATRIA study stroke risk score. J Am Heart Assoc. 2013;2(3):1-10. https://doi.org/10.1161/JAHA.113.000250

12. Wolsk E, Lamberts M, Morten LH, Blanche P, Køber L, Torp-Pedersen C, et al. Thromboembolic risk stratification of patients hospitalized with heart failure in sinus rhythm: a nationwide cohort study. Eur J Heart Fail. 2015;17(8):828-36. https://doi.org/10.1002/ejhf.309

13. Mazzone C, Cioffi G, Carriere C, Barbati G, Faganello G, Russo G, et al. Predictive role of CHA2DS2-VASc score for cardiovascular events and death in patients with arterial hypertension and stable sinus rhythm. Eur J Prev Cardiol. 2017;24(15):1584-93 https://doi.org/10.1177/2047487317726068

14. Guichard JB, Nattel S. Atrial cardiomyopathy: A useful notion in cardiac disease management or a passing fad? J Am Coll Cardiol. 2017;70:756-65. https://doi.org/10.1016/j.jacc.2017.06.033

15. Kottkamp H. Fibrotic atrial cardiomyopathy: A specific disease/ syndrome supplying substrates for atrial fibrillation, atrial tachycardia, sinus nodedisease, AVnodedisease, and thromboembolic complications. J Cardiovasc Electrophysiol. 2012;23(7):789-99. https:// doi.org/10.1111/j.1540-8167.2012.02341.x

16. Goette A, Kalman JM, Aguinaga L, Akar J, Cabrera JA, Chen SA, et al. EHRA/HRS/APHRS/SOLAECE expert consensus on atrial cardiomyopathies: definition, characterization, and clinical implication. Europace. 2016;18(10):1455-90. https://doi. org/10.1093/europace/euw161

17. Froehlich L, Meyre P, Aeschbacher S, Blum S, Djokic D, Kuehne $\mathrm{M}$, et al. Left atrial dimension and cardiovascular outcomes in patients with and without atrial fibrillation: a systematic review and meta-analysis. Heart. 2019;105(24):1884-91. https://doi. org/10.1136/heartjnl-2019-315174

18. Daccarett M, Badger TJ, Akoum N, Burgon NS, Mahnkopf C, Vergara $G$, et al. Association of left atrial fibrosis detected by delayed-enhancement magnetic resonance imaging and the risk of stroke in patients with atrial fibrillation. J Am Coll Cardiol. 2011;57(7):831-8. https://doi.org/10.1016/j.jacc.2010.09.049

19. Akoum N, Fernandez G, Wilson B, Mcgann C, Kholmovski E, Marrouche N. Association of atrial fibrosis quantified using LGE-MRI with atrial appendage thrombus and spontaneous contrast on transesophageal echocardiography in patients with atrial fibrillation.J Cardiovasc Electrophysiol. 2013;24(10):1104-9. https://doi.org/10.1111/jce.12199

20. King JB, Azadani PN, Suksaranjit P, Bress AP, Witt DM, Han $\mathrm{FT}$, et al. Left atrial fibrosis and risk of cerebrovascular and cardiovascular events in patients with atrial fibrillation. J Am Coll Cardiol. 2017;70(11):1311-21. https://doi.org/10.1016/j. jacc.2017.07.758

21. Müller $P$, Makimoto $H$, Dietrich JW, Fochler $F$, Nentwich $K$, Krug J, et al. Association of left atrial low-voltage area and thromboembolic risk in patients with atrial fibrillation. Europace. 2018;20(3):f359-65. https://doi.org/10.1093/europace/eux172

22. Glotzer TV, Daoud EG, Wyse DG, Singer DE, Ezekowitz MD, Hilker C, et al: The relationship between daily atrial tachyarrhythmia burden from implantable device diagnostics and stroke risk: the TRENDS study. Circ Arrhythm Electrophysiol. 2009;2(5):474-80. https://doi.org/10.1161/CIRCEP.109.849638

23. Daoud EG, Glotzer TV, Wyse DG, Ezekowitz MD, Hilker C, Koehler J, et al:Temporal relationship of atrial tachyarrhythmias, cerebrovascular events, and systemic emboli based on stored device data: A subgroup analysis of TRENDS. Heart Rhythm. 2011;8(9):1416-23. https://doi.org/10.1016/j.hrthm.2011.04.022

24. Brambatti M, Connolly SJ, Gold MR, Morillo CA, Capucci A, Muto C, et al: Temporal relationship between subclinical ttrial fibrillation and embolic events. Circulation. 2014;129(21):2094-99. https:// doi.org/10.1161/CIRCULATIONAHA.113.007825

25. Martin DT, Bersohn MM, Waldo AL, Wathen MS, Choucair WK, Lip GY, et al: Randomized trial of atrial arrhythmia monitoring to guide anticoagulation in patients with implanted defibrillator and cardiac resynchronization devices. Eur HeartJ. 2015;36(26):16608. https://doi.org/10.1093/eurheartj/ehv115

26. Mahajan R, Perera T, Elliott AD, Twomey DJ, Kumar S, Munwar $D A$, et al: Subclinical device-detected atrial fibrillation and stroke risk: a systematic review and meta-analysis. Eur Heart J. 2018;39(16):1407-15. https://doi.org/10.1093/eurheartj/ehx731 